\title{
Capacity of Zero-Outage Scheme Under Imprecise Channel State Information
}

\author{
Çag̃atay Candan
}

\begin{abstract}
The capacity of zero-outage scheme with imprecise channel state information at the transmitter side (CSIT) for frequency flat, single-input multiple-output (SIMO) channels is examined. It is shown that when CSIT is not precise, the receiver signal-to-noise-ratio fluctuates and the scheme suffers from communication outages. Exact analytical expressions characterizing the outage capacity, the additional power required to compensate the effect of noisy CSIT are given and the impact of noisy CSIT on the ergodic capacity is examined.
\end{abstract}

Index Terms-Zero-outage scheme, outage capacity, ergodic capacity, imprecise CSI.

\section{INTRODUCTION}

$\mathbf{I}$ $\mathrm{T}$ is well known that the presence of precise channel state information at the transmitter side (CSIT) enables the system designer to convert a fading channel to a non-fading channel with a procedure called channel inversion, [1, p.111]. The conversion to the non-fading condition is achieved by adjusting the instantaneous transmit power so as to compensate the channel conditions such that the receiver signal-to-noiseratio (SNR) is constant for all fading states under a long term average transmit power constraint. Even though this scheme is not optimal in the sense of maximizing the channel capacity, its relative simplicity and the availability of capacity achieving codes for additive white noise channels makes this scheme attractive. The capacity of the described scheme is denoted as the zero-outage capacity in the literature, [1], [2], [3], [4].

In this letter, we examine the effect of impresice CSIT on the zero-outage capacity. It should be clear that the imperfections in CSIT prohibit the transmitter to exactly compensate the channel state and achieve a constant SNR at the receiving end. This letter examines the SNR fluctuation at the receiver due to noisy CSIT and its effect on the capacity. The SNR fluctuation can also be interpreted as a type of fading due to imperfect knowledge of the channel state. In the present work, we examine the zero-outage capacity for single user, flat fading single-input multiple-output (SIMO) Rayleigh channels. The capacity with perfect CSIT for independent Rayleigh fading channels with $D$ receivers ( $D$ is also the diversity level) is given as $\log _{2}\left(1+\frac{D-1}{D} \frac{P_{a v}}{\sigma_{n}^{2}}\right)$ bits/sec/Hz [3, eq. (3.3.30)]. Our goal is to characterize the capacity of the same system under noisy CSIT.

It should be remarked that the phrase "zero-outage" does not describe the operation of the scheme under imprecise CSIT. Yet, we use "zero-outage capacity with imprecise CSIT" and

Manuscript received October 11, 2012. The associate editor coordinating the review of this letter and approving it for publication was T. Tsiftsis.

The authors is with the Department of Electrical-Electronics Engineering, METU, Ankara, Turkey (e-mail: ccandan@metu.edu.tr).

Digital Object Identifier 10.1109/LCOMM.2012.120312.122275 similar phrases in order to position the present study within the related works in the literature. Related works in the literature are the initial work of Caire et al. [2] on the delay limited capacity for the block fading channels and its extension to multiple antenna systems by Biglieri et al. [5]. Sung et al. examines the delay limited capacity of MIMO-OFDM systems [6] and an extension to multi-user communication is given in [7]. To the best of our knowledge, the effect of channel estimation errors on the capacity of zero-outage scheme, that is the maximization of the mutual information given noisy CSI at transmitter, is not studied in the literature in spite of its mention by Caire et al. in [2].

\section{System Model AND PRELIMINARIES}

System model for a flat fading, quasi-static SIMO communication system with $D$ receiving antennas is written as follows:

$$
y_{k}=\sqrt{\mathrm{SNR}} h_{k} x+n_{k}, \quad k=\{1, \ldots, D\}
$$

Here $h_{k}(k=\{1, \ldots, D\})$ is the complex valued channel gain which is independent and identically distributed (i.i.d.) circularly symmetric complex Gaussian random variables with zero mean and variance $1 / D, h_{k} \sim \mathcal{C N}(0,1 / D)$; $n_{k}$ is i.i.d. receiver noise with the distribution $\mathcal{C N}(0,1)$; the data symbol $x$ is assumed to be zero mean and unit variance. The variable SNR appearing in (1) can be considered as the output SNR after the maximum ratio combining of $D$ antenna outputs.

In this paper, we assume that the channel gains are estimated through the transmission of a pilot. The minimum mean square error (MMSE) estimate of $h_{k}$ can be written as follows, [8]:

$$
\widehat{h}_{k}=\frac{\sqrt{\mathrm{SNR}_{\mathrm{T}}}}{\mathrm{SNR}_{\mathrm{T}}+D} y_{k}, \quad k=\{1, \ldots, D\}
$$

Here $\mathrm{SNR}_{\mathrm{T}}$ refers to the $\mathrm{SNR}$ at the training phase which can be greater than the operation SNR. The random variables $h_{k}$ and its estimate $\widehat{h}_{k}$ are both Gaussian distributed with the following joint distribution:

$$
\left[\begin{array}{l}
h_{k} \\
\widehat{h}_{k}
\end{array}\right] \sim \mathcal{C N}\left(\left[\begin{array}{l}
0 \\
0
\end{array}\right],\left[\begin{array}{cc}
1 / D & \rho / D \\
\rho / D & \rho / D
\end{array}\right]\right) .
$$

Here $\rho=\frac{\mathrm{SNR}_{\mathrm{T}}}{\mathrm{SNR}_{\mathrm{T}}+D}$ and $\sqrt{\rho}$ is the correlation coefficient of $h_{k}$ and $\widehat{h}_{k}$. The parameter $\rho$ is denoted as the power correlation coefficient in the literature.

The channel gains $\left(h_{k}\right)$ are independent from each other; therefore, the random variable $\|\mathbf{h}\|^{2}=\sum_{k=1}^{D}\left|h_{k}\right|^{2}$ is chisquare distributed with 2D degrees of freedom. In addition, $E\left\{\|\mathbf{h}\|^{2}\right\}$ (the power gain) is 1 . It should be noted that the chi-square distribution representing $\|\mathbf{h}\|^{2}$ is also denoted as the gamma distribution with the shape parameter $D$ and scale 
parameter $\Omega=E\left\{\|\mathbf{h}\|^{2}\right\}=1$. Similar to $\|\mathbf{h}\|^{2}$, the random variable $\|\widehat{\mathbf{h}}\|^{2}$ is gamma distributed with the same shape parameter $(D)$, but with a scale parameter of $\Omega=\rho$.

The joint distribution of the random variables $\|\mathbf{h}\|^{2}$ and $\|\widehat{\mathbf{h}}\|^{2}$ is required for the present analysis. Following the discussion presented in [9], the correlation coefficient of the random variables $\|\mathbf{h}\|^{2}$ and $\|\widehat{\mathbf{h}}\|^{2}$ can be shown to be $\rho$. The joint probability density function of $p_{1}=\|\mathbf{h}\|^{2}$ and $p_{2}=\|\widehat{\mathbf{h}}\|^{2}$ has been given in [10, eq.(5)] and presented in (4) (on the next page).

$\Gamma(\cdot)$ appearing in (4) is the gamma function, $I_{\alpha}(x)=$ $\sum_{k=0}^{\infty}(x / 2)^{\alpha+2 k} /(\Gamma(\alpha+k+1) k !)$ is the modified Bessel function of order $\alpha$ and $\mathrm{u}(\cdot)$ is the unit step function. The probability distribution given in (4) is called as the bivariate Gamma distribution and represents the joint distribution of $p_{1}=\|\mathbf{h}\|^{2}$ and $p_{2}=\|\widehat{\mathbf{h}}\|^{2}$ with the common shape parameter of $D$ and the scale parameters $\Omega_{1}=1$ and $\Omega_{2}=\rho$. As a cautionary remark, we would like to note that there are different bivariate Gamma densities generalizing the ordinary gamma density in the literature, [11, p. 438]. The one given in (4) is known as the Jensen's bivariate density in the statistics literature. This remark concludes required background on the distribution of channel gains and their estimates for the study of the zero-outage capacity under noisy CSIT.

\section{ZERO-OUTAGE CAPACITY}

The capacity for the SIMO communication channel $\mathbf{y}_{i}=$ $\mathbf{h}_{i} \sqrt{P(i)} x+\mathbf{n}$ is $\log _{2}\left(1+\left\|\mathbf{h}_{i}\right\|^{2} P(i) / \sigma^{2}\right)$ bits/sec/Hz for a fixed vector of channel gains $\mathbf{h}_{i}$. Here $P(i)$ is the allocated transmitter power at time $i, \mathbf{h}_{i}$ is a $D \times 1$ complex valued vector formed by concatenating the individual channel gains and $\sigma^{2}$ is the noise variance at the receiver side.

The channel capacity for random channel gains is a random variable. One way of combating the randomness of the channel is the allocation of the transmitter power $P(i)$ such that the receiver SNR, SNR $=\|\mathbf{h}\|^{2} P(i) / \sigma^{2}$, is constant for all channel states. This scheme is called as the zero-outage scheme in the literature, [1, p.111].

Assuming the availability of precise channel information at the transmitter, the instantaneous power is set as $P(i)=\frac{\bar{P}}{\left\|\mathbf{h}_{i}\right\|^{2} E\left\{1 /\left\|\mathbf{h}_{i}\right\|^{2}\right\}}$ in the zero-outage scheme. It should be noted that the stated power allocation satisfies the long term transmitter power constraint of $E\{P(i)\}=\bar{P}$, where $\bar{P}$ is the available average power at the transmitter. With the given power allocation, the system model becomes $\mathbf{y}_{i}=\frac{\mathbf{h}_{i}}{\left\|\mathbf{h}_{i}\right\|} \sqrt{\frac{\bar{P}}{E\left\{1 /\left\|\mathbf{h}_{i}\right\|^{2}\right\}}} x+\mathbf{n}$ and the receiver SNR becomes $\frac{P}{\sigma^{2} E\left\{1 /\|\mathbf{h}\|^{2}\right\}}$, which is a non-random constant. In effect, with the zero-outage scheme the SIMO fading channel is converted to an AWGN channel with the capacity of $\log _{2}\left(1+\frac{\bar{P}}{\sigma^{2} E\left\{1 / \|\left.\mathbf{h}\right|^{2}\right\}}\right)$ bits/sec/Hz. Further details of the scheme is illustrated with several examples in [1].

When CSIT is not precise, the instantaneous power is set according to the distribution of $\widehat{\mathbf{h}}$ (instead of $\mathbf{h}$ ), $P(i)=$ $\frac{\bar{P}}{\left\|\widehat{\mathbf{h}}_{i}\right\|^{2} E\left\{1 /\left\|\widehat{\mathbf{h}}_{i}\right\|^{2}\right\}}$. Under noisy CSIT conditions, the system model with the given power allocation becomes as follows:

$$
\mathbf{y}_{i}=\underbrace{\frac{\mathbf{h}_{i}}{\left\|\widehat{\mathbf{h}}_{i}\right\|}}_{\mathbf{a}_{i}} \underbrace{\sqrt{\frac{\bar{P}}{E\left\{1 /\left\|\widehat{\mathbf{h}}_{i}\right\|^{2}\right\}}}}_{b} x+\mathbf{n}=\mathbf{a}_{i} b x+\mathbf{n}
$$

It should be noted that $\mathbf{a}_{i}$ and $b$ given in (5) is a random vector and a non-random scalar, respectively. With this observation, the capacity of the channel $\mathbf{y}_{i}=\mathbf{a}_{i} b x+\mathbf{n}$ can be immediately written as, $\log _{2}\left(1+\left\|\mathbf{a}_{i}\right\|^{2} b^{2} / \sigma^{2}\right)=$ $\log _{2}\left(1+\frac{\|\mathbf{h}\|^{2}}{\|\underline{\mathbf{h}}\|^{2}} \frac{\bar{P}}{E\left\{1 /\|\widehat{\mathbf{h}}\|^{2}\right\} \sigma^{2}}\right)$. Here $\frac{\|\mathbf{h}\|^{2}}{\|\widehat{\mathbf{h}}\|^{2}} \frac{\bar{P}}{E\left\{1 /\|\widehat{\mathbf{h}}\|^{2}\right\} \sigma^{2}}$ is a random variable representing the receiver SNR. We note that this random variable is related to the ratio of correlated bivariate gamma variates $\|\mathbf{h}\|^{2}$ and $\|\widehat{\mathbf{h}}\|^{2}$ whose joint density is given in (4).

The density for the ratio of correlated gamma variates, $r=$ $p_{1} / p_{2}$ where $p_{1}=\|\mathbf{h}\|^{2}$ and $p_{2}=\|\widehat{\mathbf{h}}\|^{2}$, can be written as follows, [12, eq. (10) $]^{1}$ :

$f_{r}(r)=\frac{2^{2 D-1}}{\sqrt{\pi}} \frac{\Gamma(D+1 / 2)}{\Gamma(D)} \frac{(1-\rho)^{D} \lambda^{D} r^{D-1}(r+\lambda)}{\left[(r+\lambda)^{2}-4 \rho \lambda r\right]^{D+0.5}} u(r)$

Here $\lambda$ is the ratio of average powers, $\lambda=\Omega_{1} / \Omega_{2}=$ $1 / \rho=\left(\mathbf{S N R}_{\mathrm{T}}+D\right) / \mathrm{SNR}_{\mathrm{T}}$. Denoting the random variable $\|\mathbf{h}\|^{2} /\|\widehat{\mathbf{h}}\|^{2}$, whose density is given in (6), with $r$ and making use of $E\left\{1 /\|\widehat{\mathbf{h}}\|^{2}\right\}=\frac{D}{\rho(D-1)}$; the receiver SNR $\frac{\|\mathbf{h}\|^{2}}{\|\widehat{\mathbf{h}}\|^{2}} \frac{\bar{P}}{E\left\{1 /\|\widehat{\mathbf{h}}\|^{2}\right\} \sigma^{2}}$ can be compactly expressed as $\frac{\bar{P}}{\sigma^{2}} \frac{D-1}{D} \rho r$. Then, the channel capacity for a fixed $r$ becomes $\log _{2}(1+$ $\left.\frac{\bar{P}}{\sigma^{2}} \frac{D-1}{D} \rho r\right) \mathrm{bits} / \mathrm{sec} / \mathrm{Hz}$. Since the density of $r$ is analytically available from (6), the capacity characterization of the zerooutage scheme under noisy CSIT is completed. In the following sections, we present the details on the ergodic and outage capacity calculations.

\section{A. Ergodic Capacity}

The ergodic capacity is the mean of the random variable $\log _{2}\left(1+\frac{\bar{P}}{\sigma^{2}} \frac{D-1}{D} \rho r\right), E_{r}\left\{\log _{2}\left(1+\frac{\bar{P}}{\sigma^{2}} \frac{D-1}{D} \rho r\right)\right\}$. By making use of the relation $\log (1+x) \approx \log (x)$, the ergodic capacity can be approximated as $E_{r}\left\{\log _{2}\left(\frac{\bar{P}}{\sigma^{2}} \frac{D-1}{D} \rho r\right)\right\}$ at high SNR conditions, that is

$$
C_{\text {ergodic }} \approx \log _{2}\left(\frac{\bar{P}}{\sigma^{2}} \frac{D-1}{D}\right)+\log _{2}(\rho)+E\left\{\log _{2}(r)\right\} .
$$

From (7), we can note that the calculation of ergodic capacity for the presented scheme reduces to the evaluation $E\left\{\log _{2}(r)\right\}$. For the evaluation of this expected value operation, the generalized moment function of $r, \Phi_{r}(s)=E\left\{r^{s}\right\}$ for a non-negative real valued $s$, can be utilized. The function $\Phi_{r}(s)(s<D)$ can be expressed as follows, [13, Corollary 1]:

$$
\Phi_{r}(s)=\left(\frac{\Omega_{1}}{\Omega_{2}}\right)^{s} \underbrace{\frac{\Gamma(D+s) \Gamma(D-s)}{\Gamma(D) \Gamma(D)}{ }_{2} F_{1}(-s, s ; D ; \rho)}_{g(s)}
$$

Here ${ }_{2} F_{1}(\cdot)$ denotes the generalized hypergeometric function. By evaluating the first derivative of $\Phi_{r}(s)$ at $s=0$, we can get

\footnotetext{
${ }^{1}$ Equation (6) is the corrected version of [12, eq. (10)] where the factor of $\lambda^{D}$ is missing.
} 


$$
f_{p_{\mathrm{k}} p_{2}}\left(p_{1}, p_{2}\right)=\frac{D^{D+1}}{\Gamma(m) \Omega_{1} \Omega_{2}(1-\rho)}\left(\frac{p_{1} p_{2}}{\rho \Omega_{1} \Omega_{2}}\right)^{\frac{D-1}{2}} \exp \left\{-\frac{D}{1-\rho}\left(\frac{p_{1}}{\Omega_{1}}+\frac{p_{2}}{\Omega_{2}}\right)\right\} I_{D-1}\left(\frac{2 D}{1-\rho} \sqrt{\rho \frac{p_{1} p_{2}}{\Omega_{1} \Omega_{2}}}\right) \mathrm{u}\left(p_{1}\right) \mathrm{u}\left(p_{2}\right)
$$

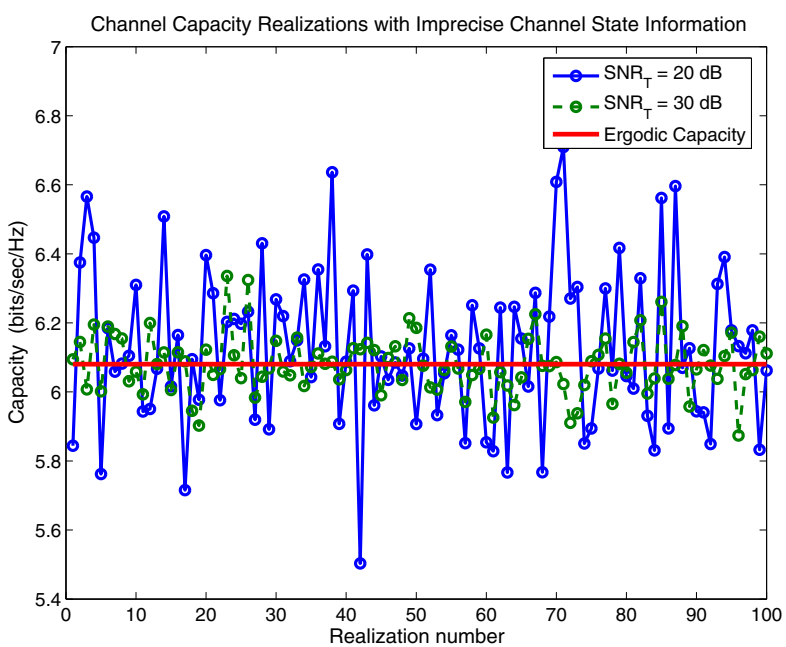

Fig. 1. A set of realizations for the zero-outage scheme with $D=3$ receiving antennas under imprecise CSIT conditions.

$\left.\frac{d}{d s} \Phi_{r}(s)\right|_{s=0}=E_{r}\{\ln (r)\}$. Noting that $\Phi_{r}(s)=\left(\frac{\Omega_{1}}{\Omega_{2}}\right)^{s} g(s)$ and the evenness of the function $g(s)$, the first derivative of $\Phi_{r}(s)$ at $s=0$ can be easily calculated as $\ln \left(\Omega_{1} / \Omega_{2}\right)$. With the substitution of $\Omega_{1}=1$ and $\Omega_{2}=\rho$, we can finalize the evaluation of expected value operation, $E\left\{\log _{2}(r)\right\}=$ $-\log _{2}(\rho)$.

Upon the substitution of $E\left\{\log _{2}(r)\right\}=-\log _{2}(\rho)$ into (7), the ergodic capacity under noisy CSIT becomes $\log _{2}\left(\frac{\bar{P}}{\sigma^{2}} \frac{D-1}{D}\right)$. It should be noted that this is exactly the value of the ergodic capacity with precise CSIT! This surprising result can be explained by noting that the loss term in equation (7), which is $\log _{2}(\rho)$, is perfectly cancelled with the term $E\left\{\log _{2}(r)\right\}$ related to the fluctuation of receiver SNR.

Fig. 1 is presented to examine this surprising result in more detail. Here 100 capacity realizations are shown for a system with $D=3$ receiving antennas and operating at the SNR of $\bar{P} / \sigma^{2}=20 \mathrm{~dB}$. The channel coefficients are estimated at $\mathrm{SNR}_{\mathrm{T}}=\{20,30\} \mathrm{dB}$. The presented analytical result states that the average of realizations for different $\mathrm{SNR}_{\mathrm{T}}$ values are identical which is confirmed by Monte Carlo experiments.

Fig. 1 illustrates that the fluctuation around the mean value, that is fluctuation around ergodic capacity valve, is much more significant for lower training SNR values. It is possible to conclude from this figure that the main cost of poor CSI is not a reduction in the ergodic capacity; but the fluctuation of the instantaneous capacity. In the next section, we examine this fluctuation and its effect on the outage capacity.

\section{B. Outage Capacity}

The communication outages occur when the instantaneous capacity falls below the information rate. For the examined scheme, the probability of this event is $P_{\text {out }}(R)=P\left\{\log _{2}(1+\right.$ $\left.\left.\left\|\mathbf{a}_{i}\right\|^{2} b^{2} / \sigma^{2}\right)<R\right\}$ where $R$ is the information rate and the

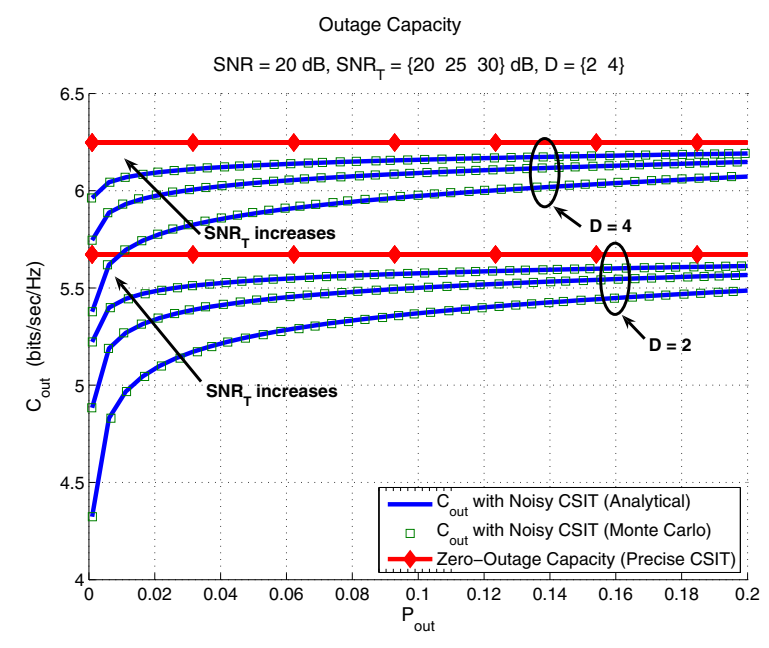

Fig. 2. Outage capacity of the described scheme at different SNR, training SNR and diversity levels.

definitions for $\mathbf{a}_{i}$ and $b$ are given in (5). Using the density of $r=\left\|\mathbf{a}_{i}\right\|^{2}=\frac{\|\mathbf{h}\|^{2}}{\|\widehat{\mathbf{h}}\|^{2}}$, given in (6), the outage probability can be expressed as $P_{\text {out }}(R)=F_{r}\left(\sigma^{2}\left(2^{R}-1\right) / b^{2}\right)$ where $F_{r}(z)=\int_{0}^{z} f_{r}(x) d x$ is the cumulative distribution function (c.d.f.) of the random variable $r$. Equivalently, the maximum information rate that can carried at an outage probability of $P_{\text {out }}$ is

$$
C_{\text {out }}=\log _{2}\left(1+F_{r}^{-1}\left(P_{\text {out }}\right) b^{2} / \sigma^{2}\right) \quad \text { bits/sec/Hz. }
$$

The cumulative distribution function $F_{r}(r)$ appearing in the outage relations is a complicated function to analytically express for the generic values of $D$ and $\lambda$. Yet, the same function can be easily written for a given set of numerical values. For example, the distribution function $F_{r}(r)$ for $D=2$ and $\lambda=1.2$ (corresponds to $\mathrm{SNR}_{\mathrm{T}}=10 \mathrm{~dB}$ ) can be expressed as follows:

$$
F_{r}(r)=\frac{1}{2}\left(1+\frac{(5 r-6)\left(25 r^{2}-30 r+36\right)}{\left(25 r^{2}-40 r+36\right)^{3 / 2}} u(r)\right)
$$

Similar analytical expressions for $F_{r}(r)$ can be derived using standard integration techniques when numerical values for $D$ and $\lambda$ are given.

Fig. 2 shows the outage capacity for the operational SNR $\left(\bar{P} / \sigma^{2}\right)$ of $20 \mathrm{~dB}$, the training SNR values of $\{20,25,30\} \mathrm{dB}$ and $D=\{2,4\}$. The capacity of the system with precise CSIT is also provided as an upper bound for the noisy CSIT cases. From this figure, we can conclude that when the training SNR is greater than operational SNR, the capacity degradation due to imprecise CSIT can be considered acceptable, say, for a $5 \%$ communication outage rate.

In Fig. 3, the amount of additional power required to operate at the capacity value of the perfect CSIT case is shown. The additional power can be considered as a penalty paid by the transmitter for the imprecise CSIT. For the calculation of this 

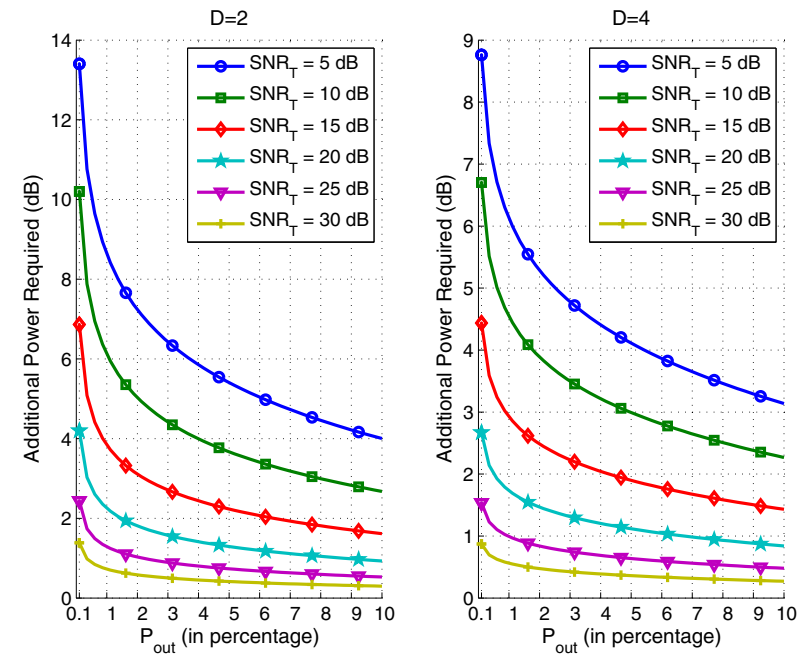

Fig. 3. Additional power required to compensate the effect of imprecise CSIT.

penalty, note that $b^{2}$ appearing in the outage capacity relation (9) can be written as $b^{2}=\frac{\bar{P}}{E\left\{1 / \| \widehat{\mathbf{h}}||^{2}\right\}}=\frac{\bar{P}}{E\left\{1 / \| \mathbf{h}||^{2}\right\}} \rho$ using (5). When $b^{2}$ is substituted into the outage capacity relation, we get $C_{\text {out }}=\log _{2}\left(1+\frac{\bar{P}}{\sigma^{2} E\left\{1 /\|\mathbf{h}\|^{2}\right\}} F_{r}^{-1}\left(P_{\text {out }}\right) \rho\right)$. It should be clear that by increasing $\bar{P}$ by $1 /\left[F_{r}^{-1}\left(P_{\text {out }}\right) \rho\right]$, we can cancel the term $F_{r}^{-1}\left(P_{\text {out }}\right) \rho$ appearing in $C_{\text {out }}$. Hence, an additional power of $-10 \log _{10}\left(F_{r}^{-1}\left(P_{\text {out }}\right) \rho\right) \mathrm{dB}$ is required to compensate the effect of noisy CSIT. Fig. 3 shows the required power for different outage probability values. An important point that should be emphasized is that both the capacity loss and the additional power required for its compensation do not depend on the operational SNR; but only depends on the "quality" of channel estimates generated by the training.

\section{Conclusions}

This paper examines the capacity degradation of the zerooutage scheme due to imprecise CSIT. It is analytically shown that the ergodic capacity of the scheme is not affected by the imprecise channel state information. The outage capacity is indeed significantly affected by the quality of CSIT. The amount of additional power compensating this degradation is analytically given. This work can be relatively easily extended to the frequency selective fading channels by following the approach given in [6]. A more difficult extension is the examination of the capacity when both receiver CSI (CSIR) and transmitter CSI is noisy. The results of this paper can be considered as an upper bound for this more difficult problem.

\section{REFERENCES}

[1] A. Goldsmith, Wireless Communications. Cambridge University Press, 2005.

[2] G. Caire, G. Taricco, and E. Biglieri, "Optimum power control over fading channels," IEEE Trans. Inf. Theory, vol. 45, no. 5, pp. 14681489, July 1999.

[3] E. Biglieri, J. Proakis, and S. Shamai, "Fading channels: informationtheoretic and communications aspects," IEEE Trans. Inf. Theory, vol. 44, no. 6, pp. 2619-2692, Oct. 1998.

[4] E. Jorswieck and H. Boche, "Delay-limited capacity: multiple antennas, moment constraints, and fading statistics," IEEE Trans. Wireless Commun., vol. 6, no. 12, pp. 4204-4208, Dec. 2007.

[5] E. Biglieri, G. Caire, and G. Taricco, "Limiting performance of blockfading channels with multiple antennas," IEEE Trans. Inf. Theory, vol. 47, no. 4, pp. 1273-1289, May 2001.

[6] J. H. Sung and J. Barry, "Approaching the zero-outage capacity of MIMO-OFDM without instantaneous water-filling," IEEE Trans. Inf. Theory, vol. 54, no. 4, pp. 1423-1436, Apr. 2008.

[7] S. Hanly and D. Tse, "Multiaccess fading channels-II: delay-limited capacities," IEEE Trans. Inf. Theory, vol. 44, no. 7, pp. 2816-2831, Nov. 1998.

[8] S. M. Kay, Fundamentals of Statistical Signal Processing, Volume 1: Estimation Theory. Prentice Hall, 1993.

[9] Q. Zhang, "A decomposition technique for efficient generation of correlated Nakagami fading channels," IEEE J. Sel. Areas Commun., vol. 18, no. 11, pp. 2385-2392, Nov. 2000.

[10] T. Piboongungon, V. Aalo, C. Iskander, and G. Efthymoglou, "Bivariate generalised gamma distribution with arbitrary fading parameters," Electron. Lett., vol. 41, no. 12, pp. 709-710, June 2005.

[11] S. Kotz, N. Balakrishnan, and N. L. Johnson, Continuous Multivariate Distributions Volume 1: Models and Applications. John Wiley \& Sons, 2000.

[12] P. S. Bithas, N. C. Sagias, T. A. Tsiftsis, and G. K. Karagiannidis, "Distributions involving correlated generalized gamma variables," in Proc. 2007 Int. Conf. on Applied Stochastic Models and Data Analysis, vol. 12.

[13] C. Candan and U. Orguner, "Moment function for the ratio of correlated generalized gamma variables," Electron. Lett., submitted, July 2012. 